\title{
Effect of Erbium Nanoparticles on Optical Properties of Zinc Borotellurite Glass System
}

\author{
Azlan Muhammad Noorazlan, Halimah Mohamed Kamari, \\ Siti Shafinas Zulkefly, and Daud W. Mohamad \\ Physics Department, Faculty of Science, University Putra Malaysia (UPM), 43400 Serdang, Selangor, Malaysia \\ Correspondence should be addressed to Halimah Mohamed Kamari; halimah@science.upm.edu.my
}

Received 27 September 2013; Accepted 7 November 2013

Academic Editor: Chunyi Zhi

Copyright (c) 2013 Azlan Muhammad Noorazlan et al. This is an open access article distributed under the Creative Commons Attribution License, which permits unrestricted use, distribution, and reproduction in any medium, provided the original work is properly cited.

\begin{abstract}
Erbium nanoparticles (NPs) doped zinc borotellurite glasses have been prepared by conventional melt-quenching technique with the chemical composition $\left\{\left[\left(\mathrm{TeO}_{2}\right)_{0.70}\left(\mathrm{~B}_{2} \mathrm{O}_{3}\right)_{0.30}\right]_{1-x}(\mathrm{ZnO})_{x}\right\}_{1-y}\left(\mathrm{Er}_{3} \mathrm{O}_{2}\right)_{y}$ (where $\left.y=0.005,0.01,0.02,0.03,0.04,0.05\right)$. The structural properties of the prepared glasses were determined via X-ray diffraction (XRD) analysis and FTIR analysis. It was confirmed that the prepared glasses are amorphous. The bonding parameters of the glasses were analyzed by using FTIR analysis and were confirmed to be ionic in nature. The refractive index increases as the content of erbium NPs increases. The optical absorption spectra revealed that fundamental absorption edge shifts to longer wavelength as the content of erbium NPs increases. The value of band gap had been calculated and shown to be decreased with an increase content of erbium NPs. The Urbach energy was shown to be linearly increased with an increase content of erbium NPs oxides.
\end{abstract}

\section{Introduction}

Rare-earth doped materials have made the largest interest to develop the photonic system for various applications with regard to their advance optical properties. Erbium oxide is one of the most potential candidates among the lanthanide group in the pursuit of photonic materials research. Erbium consists of trivalent electron which is known to be advantaged to fiber amplifier applications. Nowadays, special attention is paid to explore the optical behavior of the $\mathrm{Er}^{3+}$ ions due to its emission at $1.53 \mu \mathrm{m}$, which is ideal for applications in the field of optical data transmission [1].

The choice of a suitable host matrix is important to obtain the excellent optical properties. Tellurite oxide is known to be the best candidate among the heavy metal materials because of its unique optical and electrical properties. The compatibility and solubility of tellurite based glass to the other oxide glasses are the most excellent among the heavy metals. Recently, tellurite based glass has been used in several photonic devices such as precursors for infrared fibers and windows. Formation of pure tellurite oxide glass does not occur without contributions from external element.
Borate oxide is known as the best choice to stabilize the glass formation due to its good rare earth ion solubility and hardness. The borate matrix possesses well defined gathering of $\mathrm{BO}_{3}$ triangles and $\mathrm{BO}_{4}$ tetrahedra to form stable borate groups such as diborate, triborate, and tetraborate [2]. Contributions of zinc oxide in the glass formation reduce the melting point and increases the glass forming ability. It has been reported that [3] the effect of zinc oxide decreases the optical energy gap and increase the refractive index. Zinc oxide can occupy both network forming and network modifying positions in the borate network glasses and, as a result, the physical properties of such glasses exhibit discontinuous changes, when the structural role of the cation changes [4]. Borate oxide and zinc oxide will be used as the glass stabilizer to obtain the suitable glass system.

The information of erbium nanoparticles (NPs) oxide doped glass is not being well investigated. Nanoparticles are known to have unique quantum nature which alters the materials properties. Nowadays, research on nanotechnology has given attention to enhance the properties of materials. It is strongly required to extend the research on nanoparticles doped glass to obtain the excellent properties of photonic 
devices. Hence, this research is focused on the effect of nanoparticles to the optical properties of zinc borotellurite glass system.

\section{Experimental}

The erbium nanoparticles (NPs) doped zinc borotellurite glasses with chemical composition $\left\{\left[\left(\mathrm{TeO}_{2}\right)_{0.70}\right.\right.$ $\left.\left.\left(\mathrm{B}_{2} \mathrm{O}_{3}\right)_{0.30}\right]_{1-x}(\mathrm{ZnO})_{x}\right\}_{1-y}\left(\mathrm{Er}_{3} \mathrm{O}_{2}\right)_{y}$ (where $y=0.005,0.01$, $0.02,0.03,0.04,0.05)$ were prepared by using conventional melt-quenching method. The high purity analytical grade chemicals such as erbium (III) oxide, $\mathrm{Er}_{2} \mathrm{O}_{3}$ (99.9\%, Reacton, Alfa Aesar), erbium (III) oxide nanopowder, $\mathrm{Er}_{2} \mathrm{O}_{3}$ (99.9\%, Reacton, Alfa Aesar), tellurium (IV) oxide, $\mathrm{TeO}_{2}$ (99.99\%, Puratronic, Alfa Aesar), ZnO (99.99\%, Assay, Alfa Aesar), and boron oxide $\mathrm{B}_{2} \mathrm{O}_{3}$ (99.98\%, Assay, Alfa Aesar) were used to prepare the glasses. The chemical powders of $\mathrm{Er}_{3} \mathrm{O}_{2}, \mathrm{TeO}_{2}, \mathrm{ZnO}$, and $\mathrm{B}_{2} \mathrm{O}_{3}$ were weighed by using digital weighing machine with accuracy of $\pm 0.0001 \mathrm{~g}$. All the weighed chemicals with composition of about $13 \mathrm{~g}$ were mixed thoroughly and transferred to alumina crucible. The mixtures in alumina crucible were transferred to electrical furnace and were preheated at $400^{\circ} \mathrm{C}$ for 30 minutes.

The alumina crucible was transferred to the second furnace at $900^{\circ} \mathrm{C}$ in 2 hours for the melting process. The melts were air quenched by pouring into stainless steel split molds which had been preheated at $400^{\circ} \mathrm{C}$. The molds were then annealed in the first furnace at $400^{\circ} \mathrm{C}$ for 2 hours to remove the formation of air bubbles, thermal stress and improve the mechanical strength. The prepared glasses were cooled down to room temperature by switching off the furnace. The prepared glass samples were cut at a thickness of $2 \mathrm{~mm}$ by using Isomet Buehler low speed saw machine. The sample was polished with various types of sand papers, 1500 grid, 1200 grid, and 1000 grid, to obtain flat and smooth surface.

The density and molar volume of the prepared glass samples were calculated by using the Archimedes formula. Acetone was used as the immersion liquid and digital weighing machine was used to calculate the mass of the prepared glass samples. The refractive index of the prepared glass samples was measured by using EL X-02 C high precision Eilipsometer where the wavelength of the light source is $632.80 \mathrm{~nm}$ and the angle of incident $70^{\circ}$. Optical absorption was recorded by using UV-1650 PC UV-Vis Spectrophotometer (Shimadzu) in the wavelength range $190-1100 \mathrm{~nm}$. Both refractive index and optical absorption measurement were carried out at room temperature with dark surrounding for better result. All data were analyzed automatically and controlled by a CPU. The data of measurement were calculated by using different equations for their optical energy gap and absorption coefficient.

\section{Result and Discussion}

3.1. Density and Molar Volume. The density of glass is an important tool required for analyzing the changes in the structure of the glass system. Density was used in calculation of several important properties such as refractive index, elastic properties, and thermal conductivity. The density is
TABLE 1: Density and molar volume of erbium NPs doped zinc borotellurite glass system.

\begin{tabular}{lcc}
\hline Sample, $y$ & $\begin{array}{c}\text { Molar volume } \\
\left(\mathrm{m}^{3} / \mathrm{mol}\right)\end{array}$ & $\begin{array}{c}\text { Density } \\
\left(\mathrm{kg} / \mathrm{m}^{3}\right)\end{array}$ \\
\hline 0.000 & 32.2968 & 3.630 \\
0.005 & 25.868 & 4.588 \\
0.010 & 25.733 & 4.659 \\
0.020 & 25.771 & 4.755 \\
0.030 & 25.964 & 4.822 \\
0.040 & 26.215 & 4.877 \\
0.050 & 26.737 & 4.881 \\
\hline
\end{tabular}

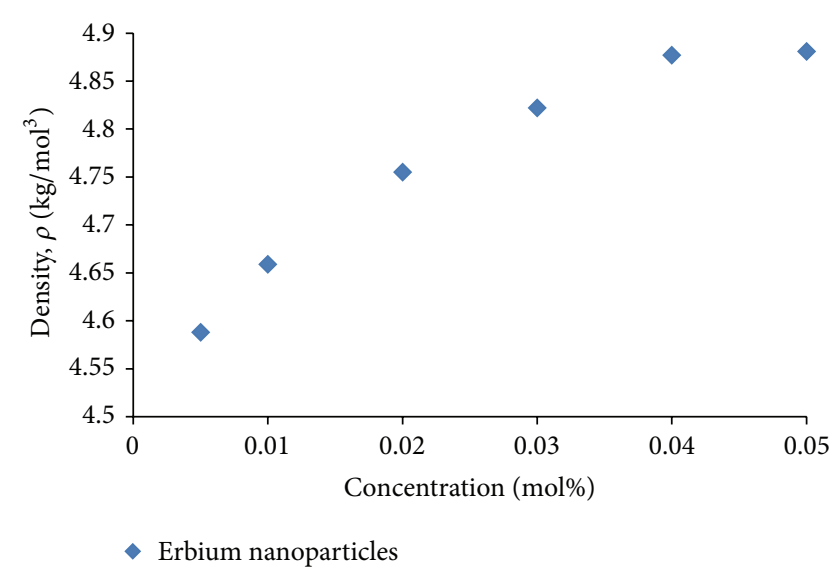

FIGURE 1: Density for erbium NPs doped zinc borotellurite glasses.

affected by the structural softening/compactness, changes in geometrical configuration, coordination number, cross-link density, and dimension of interstitial spaces of the glass [5].

The measurement data of densities of the prepared glass samples are listed in Table 1. The effect of erbium NPs on the density of zinc borotellurite glass systems is shown in Figure 1. It is observed that the density increases with an increase content of erbium NPs. The increasing value of density indicates the increasing number of nonbridging oxygen in the glass system. Erbium NPs are known to have trivalent electrons which tend to release three electrons. $\mathrm{Er}^{3+}$ ions will capture one electron from the oxygen and thus breaks the continuous network chain. Breaking the continuous network results in the formation of nonbridging oxygen ion. Each of $\mathrm{Er}^{3+}$ ions is capable to produce 3 nonbridging oxygen ions. The role of $\mathrm{Er}^{3+}$ ions increases the number of nonbridging oxygen which leads the molecules to become denser. The increasing mole fraction of $\mathrm{Er}^{3+}$ ions causes the crosslinking in the glass system to be degraded. This will result in the increasing number of density in the glass system. The existence of nanoparticles in the glass system leads the structure to become denser. Greenwood proposed that high solubility of the smaller particles causes the particles to be distributed uniformly throughout the bulk materials [6]. This will cause the structural network turn to be nearer packing and thus the density will increase. It is known that 


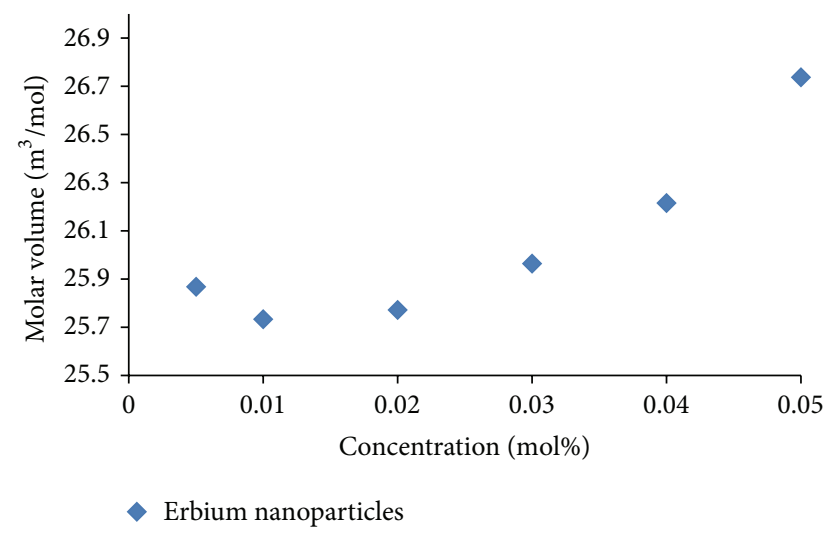

Figure 2: Molar volume for erbium NPs doped zinc borotellurite glasses.

the number of atomic mass of erbium (atomic mass of $Z_{\mathrm{Er}}=$ 167.259 $\mathrm{gmol}^{-1}$ ) is greater than the atomic mass of tellurite $\left(Z_{\mathrm{Te}}=127.6 \mathrm{gmol}^{-1}\right)$. The greater number of atomic mass in erbium compared to that in tellurite will give rise to the density in the glass system.

The molar volume data for the prepared sample is listed in Table 1. It is observed from Figure 2 that the molar volume increases with an increase content of erbium NPs. It is supposed that the relationship between density and molar volume is inverse to each other in behavior, but, in the present glass system, the relationship is different. This is due to the fact that the ionic radius of erbium NPs $(r=175 \mathrm{pm})$ is larger than that of tellurite $(r=140 \mathrm{pm})$. The increase of erbium results in the formation of excess free volume which is due to the difference of atomic radius between erbium and tellurite. Another possibility is that the behavior of $V_{m}$ is attributed to the changes in $M$ with a greater rate than that of $\rho$ [7].

\subsection{X-Ray Diffraction Analysis (XRD) and Fourier Transform} Infrared Analysis (FTIR). The XRD analysis was used to confirm the amorphous or crystalline state of the materials. The X-ray diffraction pattern of erbium NPs doped zinc borotellurite glasses was recorded in the range of $20^{\circ} \leq \theta \leq$ $80^{\circ}$. Figure 3 represents the XRD pattern of the prepared glass samples. It can be seen from Figure 3 that the XRD pattern exhibits broad diffusion at lower scattering angles indicating the presence of long range structural disorder. This confirms that the prepared glass samples are amorphous in nature and no sharp peaks are shown in the XRD pattern.

The FTIR spectroscopy is an analysis method which provides structural studies to explore the fundamental and functional groups in crystalline and non-crystalline matrices. The transmission spectra of the prepared glass samples are recorded in Figure 4 with different compositions of erbium NPs oxide. The observed broad bands are due to the combination of higher degeneracy of vibrational states, thermal broadening of the lattice dispersion, and mechanical scattering of the powdered samples and the corresponding band assignments [1]. The FTIR spectra of the prepared glass samples were recorded in the range of $400-2000 \mathrm{~cm}^{-1}$ as shown in Figure 4. It can be seen that the spectra consist of several peaks specifying their local structure. The peaks positions and their assignments are presented in Table 2 . The transmission spectra of the glass system consist of three wide absorption bands $656-664 \mathrm{~cm}^{-1}, 1233-1252 \mathrm{~cm}^{-1}$, and $1327-$ $1343 \mathrm{~cm}^{-1}$.

Tellurite oxide consists of two types of structural configuration units, that is, trigonal bipyramid, $\mathrm{TeO}_{4}$ and trigonal pyramid, $\mathrm{TeO}_{3}$. The characteristic of pure $\mathrm{TeO}_{2}$ glass was centered at $640 \mathrm{~cm}^{-1}$. The absorption band at $600-700 \mathrm{~cm}^{-1}$ was assigned by stretching vibrations of $\mathrm{Te}-\mathrm{O}$ bonds in trigonal bipyramid, $\mathrm{TeO}_{4}$ and trigonal pyramid, $\mathrm{TeO}_{3}$. Stretching vibrations of $\mathrm{TeO}_{3}$ group possess higher frequency position than that of $\mathrm{TeO}_{4}$ group. The first group of band formed around $600-650 \mathrm{~cm}^{-1}$ which corresponds to $\mathrm{TeO}_{4}$ trigonal bipyramid and the second group of band observed around $650-700 \mathrm{~cm}^{-1}$ which is due to the $\mathrm{TeO}_{3}$ trigonal pyramid [2]. The band shift of these groups depends on the changes in the composition of the glass network [3]. The appearance band at $656-664 \mathrm{~cm}^{-1}$ indicates that $\mathrm{TeO}_{3}$ group exists in all tellurite containing glass system. It can be seen that the band of $\mathrm{ZnO}$ does not appear in the spectra which means the zinc lattice is completely broken down.

The absorption band of pure borate glass, $\mathrm{B}_{2} \mathrm{O}_{3}$, was centered at $806 \mathrm{~cm}^{-1}$ frequency which indicates the characteristic of boroxyl ring. This band disappears during the glass formation which means there is no boroxyl ring in the glass system. Meanwhile, the $\mathrm{BO}_{3}$ and $\mathrm{BO}_{4}$ appear in the absorption spectra after the glass formation which is due to the substitution of boroxyl ring. The absorption spectra of the borate glass can be divided into three regions: (1) 600$800 \mathrm{~cm}^{-1}$ (bending vibrations of various borate arrangements B-O-B), (2) 800-1200 $\mathrm{cm}^{-1}$ (B-O stretching of tetrahedral $\mathrm{BO}_{4}$-units), and (3) $1200-1800 \mathrm{~cm}^{-1}$ (B-O stretching of trigonal $\mathrm{BO}_{3}$ units). The observed spectra for the first group of band are at $1233-1253 \mathrm{~cm}^{-1}$ which corresponds to B$\mathrm{O}$ (B) stretching vibrations of polymerized $\mathrm{BO}_{3}$ groups. The second group of band was observed at $1327-1343 \mathrm{~cm}^{-1}$ associated with the trigonal $\mathrm{B}-\mathrm{O}$ bond stretching vibrations in isolated trigonal $\mathrm{BO}_{3}$ units. In addition, the absorption band positioned at $1200-1253 \mathrm{~cm}^{-1}$ is assigned to the trigonal $\mathrm{B}-\mathrm{O}$ bond stretching vibrations of $\mathrm{BO}_{3}$ units from boroxyl groups. Meanwhile, the absorption band positioned at 1388$1410 \mathrm{~cm}^{-1}$ corresponds to trigonal $\mathrm{B}-\mathrm{O}$ bond stretching vibrations of $\mathrm{BO}_{3}$ units from varied types of borate groups. The absorption spectra of erbium NPs vanished during the glass formation which may be due to the fact that the low concentration of erbium NPs could not be detected by the instrument.

3.3. Refractive Index and Optical Absorption. Refractive index is the fundamental measurement carried out to determine the compatibility of the materials to be applicable in optical devices. Glasses have good compatibility with the optical properties because of the unique structural and intensity distribution. The presence of nanoparticles in the materials gives advantage to the transmission spectra of the materials. 


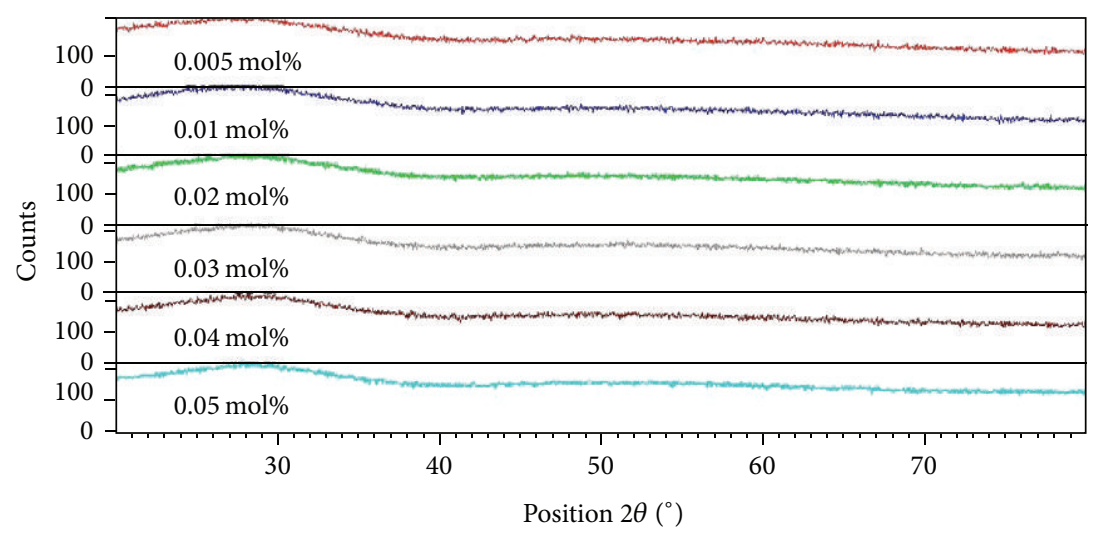

FIGURE 3: XRD patterns with different concentration of erbium NPs doped glass samples.

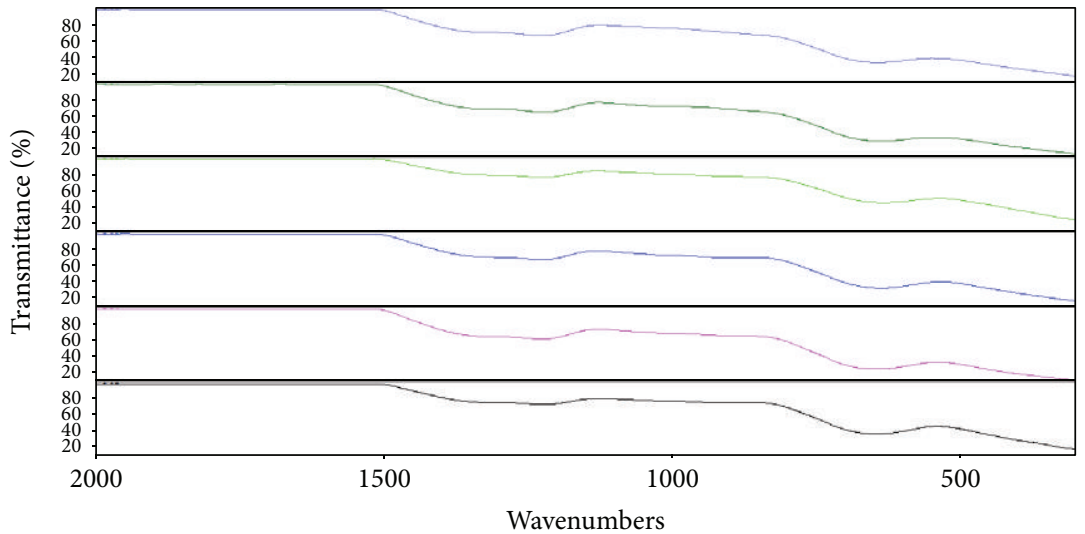

FIGURE 4: FTIR spectra of zinc oxide, boron oxide, tellurite oxide, erbium NPs oxide and $\left\{\left[\left(\mathrm{TeO}_{2}\right)_{70}\left(\mathrm{~B}_{2} \mathrm{O}_{3}\right)_{30}\right]_{70}\left(\mathrm{ZnO}_{30}\right\}_{100-y}\left(\mathrm{Er}_{3} \mathrm{O}_{2}\right)_{y}\right.$ glass system.

Factors which affect refractive index are (i) polarizability of the first neighbor ions coordinated with it (anion), (ii) coordination number of the ion (iii), electronic polarizability of the oxide ion, and (iv) optical basicity of the glasses [8]. Refractive index data for erbium NPs doped glass samples are listed in Table 3 and shown in Figure 5. The refractive index of erbium NPs doped glass samples is found in the range of 1.774-1.924. It can be seen that the refractive index increases with an increase content of erbium NPs. The results revealed that the refractive index depends on the density of the glass materials. The addition of rare earth ions $\left(\mathrm{Er}^{3+}\right)$ to the network leads to the formation of dense packing of the rare earth modifiers into the host materials [9]. This is attributed to the directly proportional behavior between refractive index and the dense packing. Higher amount of dense packing results in the higher number of refractive index of the glass system.

Trivalent erbium ions, $\mathrm{Er}^{3+}$, possess high polarity which tends to form nonbridging oxygen. Each of erbium ions will break the bridging oxygen and thus will increase the nonbridging oxygen. Formation of nonbridging oxygen will increase the polarizability of the materials. Nonbridging oxygen possesses higher polarizability than bridging oxygen. The relationship between polarizability and refractive index is a direct proportional behavior which means higher polarizability results in higher refractive index of the glass system. The presence of highly polarizable $\mathrm{Er}^{3+}$ ions with empty unfilled d-orbital (outer electronic configuration: $5 \mathrm{~d}^{0}$ $\left.6 s^{2}\right)$ contributes strongly to linear polarizability. Erbium ions, $\mathrm{Er}^{3+}$, have cation polarizability that is equal to $2.253 \mathrm{~A}^{3}$ [10] which is also strongly polarizable. Cation polarizability of erbium ions is much higher than that of tellurite $\left(1.595 \mathrm{~A}^{3}\right)$, zinc $\left(0.283 \mathrm{~A}^{3}\right)$, and borate $\left(0.002 \mathrm{~A}^{3}\right)$ [10]. The increasing molar fraction of erbium ions with higher cations polarizability leads to an increase in refractive index.

A photon with a certain range of energy can be absorbed by modifying ions in an oxide glass via either two processes: (i) internal transition between the d-shell electrons or (ii) transfer of an electron from a neighboring atom to the modifier ion and vice versa [11]. The absorption coefficient near the absorption edge can be calculated by the following formula:

$$
\alpha(\lambda)=2.303 \frac{A}{d},
$$

where $A$ denotes the absorbance and $d$ corresponds to the thickness of the glass samples. The optical absorption spectra of the prepared glass samples are shown in Figure 6. The 
TABLE 2: Assignment of infrared transmission bands of erbium NPs doped glass samples.

\begin{tabular}{lccccccc}
\hline No. & 0.005 & 0.01 & 0.02 & 0.03 & 0.04 & 0.05 & Assignments \\
\hline 1 & 1348 & 1345 & 1334 & 1341 & 1338 & 1331 & Trigonal B-O bond stretching vibrations in isolated trigonal $\mathrm{BO}_{3}$ units [5] \\
2 & 1226 & 1223 & 1225 & 1227 & 1226 & 1223 & Trigonal B-O bond stretching vibrations of $\mathrm{BO}_{3}$ units from boroxyl groups [5] \\
3 & 642 & 637 & 633 & 637 & 645 & 645 & $\mathrm{TeO}_{3}$ group exists in all tellurite containing glass [5] \\
4 & - & - & - & - & - & - & $\mathrm{ZnO}_{\text {participates in the glass network with } \mathrm{ZnO}_{4} \text { structural units and alternate TeO }}$ units [5] \\
\hline
\end{tabular}

TABLE 3: Refractive index of erbium NPs doped zinc borotellurite glass system.

\begin{tabular}{lc}
\hline Sample, $y$ & Refractive index, $n$ \\
\hline 0.000 & 1.7000 \\
0.005 & 1.7740 \\
0.010 & 1.8180 \\
0.020 & 1.8300 \\
0.030 & 1.8550 \\
0.040 & 1.8780 \\
0.050 & 1.9239 \\
\hline
\end{tabular}

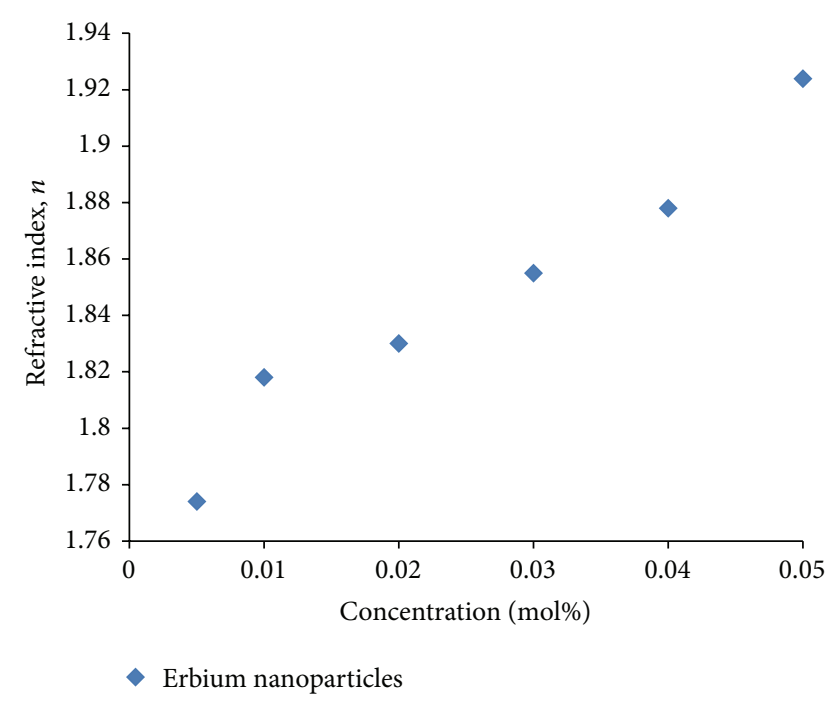

Figure 5: Refractive index of erbium NPs doped glass samples.

absorption spectra of the prepared glass samples consist of five bands of $\mathrm{Er}^{3+}$ ions at 487.0; 521.5; 545.5; 652.0; and $920.5 \mathrm{~nm}$ wavelengths. This corresponds to the absorption from the ground state ${ }^{5} \mathrm{I}_{8}$ to the excited states ${ }^{4} \mathrm{~F}_{7 / 2}+{ }^{2} \mathrm{H}_{11 / 2}$ $+{ }^{4} \mathrm{~S}_{3 / 2}+{ }^{4} \mathrm{~F}_{9 / 2}+{ }^{4} \mathrm{I}_{9 / 2}+{ }^{4} \mathrm{I}_{11 / 2}$. The optical absorption edge extended over wide wavelength, that is, no sharp edge (Urbach edge), which indicates the amorphous nature of the prepared samples [4]. It can be seen from the figure that there is no sharp absorption edge which indicates the amorphous characteristic of glassy state. The absorption edge is affected by the oxygen bond strength in the glass system. The obtained data shows the change of the oxygen bond strength in the glass system. It is observed that the fundamental edge shifts to the longer wavelength with the increase of erbium NPs. This may be due to the less rigidity of the glass system [3].

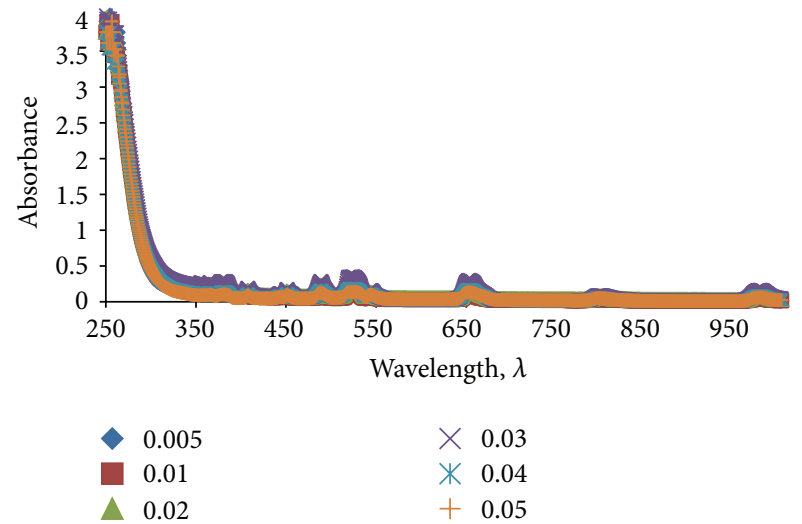

FIgURE 6: Optical absorbance spectra for erbium nanoparticles doped glasses $\left\{\left[\left(\mathrm{TeO}_{2}\right)_{0.70}\left(\mathrm{~B}_{2} \mathrm{O}_{3}\right)_{0.30}\right]_{1-x}(\mathrm{ZnO})_{x}\right\}_{1}\left(\mathrm{Er}_{3} \mathrm{O}_{2}\right)_{y}$.

3.4. Optical Band Gap and Urbach Energy. Optical absorption is an important parameter to investigate optically induced transition and to determine the structural properties and optical band gap energy. Mott and Davis proposed the relation between absorption coefficient with photon energy to calculate an indirect and direct transition occurring in a band gap [12]. The photon energy can be calculated by using the following equation:

$$
\hbar \omega=\frac{h}{2 \pi}(2 \pi f)=h f=\frac{h c}{\lambda},
$$

where $c=2.9979 \times 10^{8}(\mathrm{~m} / \mathrm{s})$

$$
\hbar=4.14 \times 10^{-15} \text { (eVs). }
$$

The absorption coefficient $\alpha(\omega)$ as a function of photon energy $\hbar \omega$ or direct and indirect optical transition as proposed by Mott and Davis is given by

$$
\alpha(\omega)=\frac{B\left(\hbar \omega-E_{\mathrm{opt}}\right)^{n}}{\hbar \omega},
$$

where $B$ is a constant related to the extent of the band tailing, $n=2$ for indirect transition, and $n=1 / 2$ for allowed direct transitions. $E_{\mathrm{opt}}$ is the optical energy gap between the valence band and conduction band. In both cases, electromagnetic waves interact with the electrons in the valance band, which are raised across the fundamental gap to the conduction band [13].

The absorption coefficient $\alpha(\omega)$ is determined near the absorption edge of all different glass samples. It is well known 


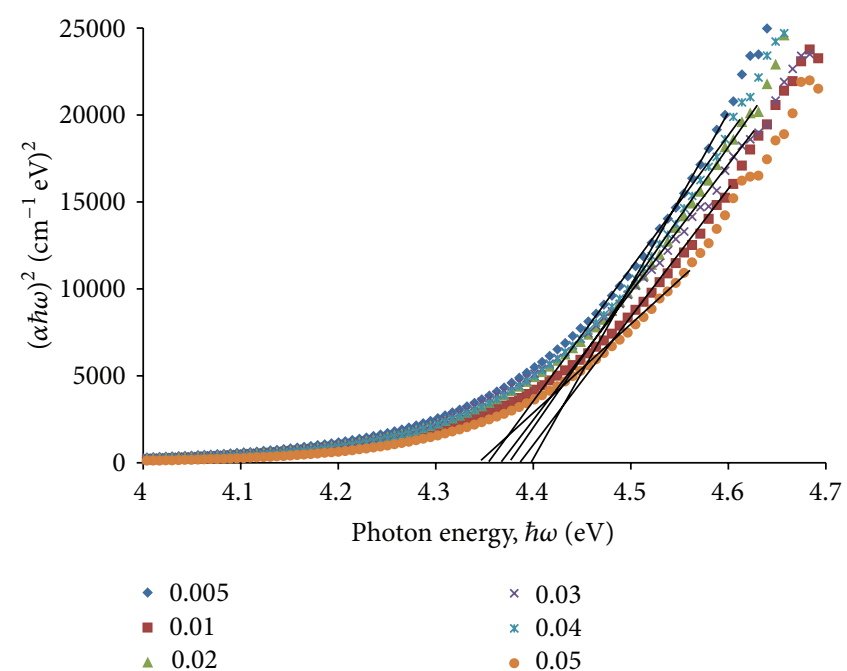

(a)

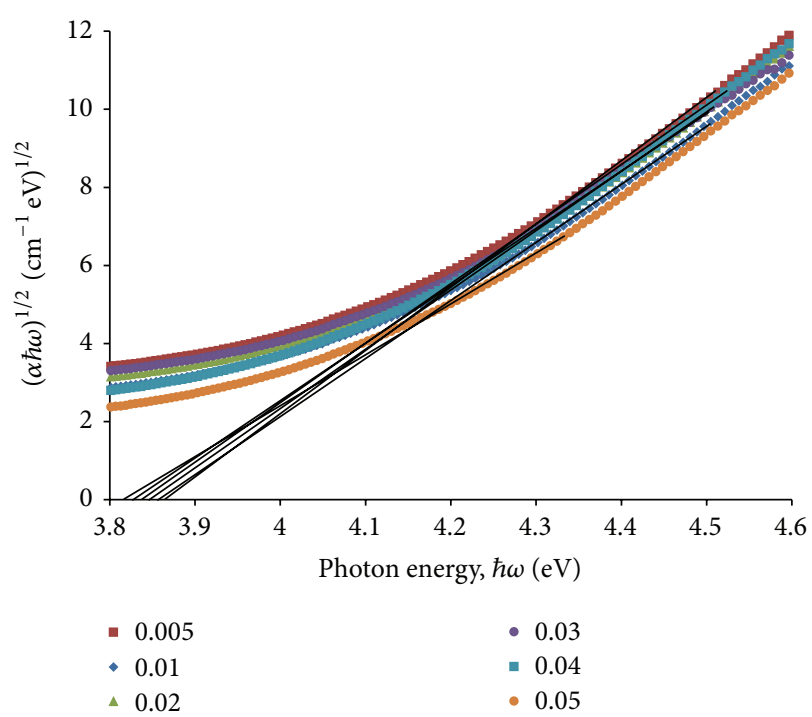

(b)

Figure 7: (a) Plot of $(\alpha \hbar \omega)^{2}$ against photon energy $\hbar \omega$ of erbium NPs doped glass samples for direct band gap measurement. (b) Plot of $(\alpha \hbar \omega)^{1 / 2}$ against photon energy $\hbar \omega$ of erbium NPs doped glass samples for indirect band gap measurement.

that, for amorphous materials, a reasonable fit to (4) with $n=2$ is achieved [13]. Therefore, (4) for amorphous material becomes

$$
(\alpha \hbar \omega)^{1 / 2}=B\left(\hbar \omega-E_{\text {opt }}\right) .
$$

In amorphous material, the plot of $(\alpha \hbar \omega)^{1 / 2}$ versus $\hbar \omega$ was also plotted for direct band gap to see whether optical data on the sample glasses fit better to direct or indirect band gap formula and (5) for $n=1 / 2$ becomes

$$
(\alpha \hbar \omega)^{2}=B\left(\hbar \omega-E_{\text {opt }}\right) .
$$

The relation in (5) is used to plot the graph of $(\alpha \hbar \omega)^{1 / 2}$ versus $\hbar \omega$. Meanwhile, (6) is used to plot $(\alpha \hbar \omega)^{2}$ versus $\hbar \omega$ for different compositions of sample glasses. The values of indirect optical band gap $E_{\text {opt }}^{1}$ and direct optical band gap $E_{\text {opt }}^{2}$ for erbium NPs doped glass samples can be obtained by extrapolating the straight line on the curve in Figures 7(a) and 7 (b) to $\hbar \omega$ axis at $(\alpha \hbar \omega)^{1 / 2}$ and $(\alpha \hbar \omega)^{2}=0$, respectively.

The higher energy parts of the spectra particularly those associated with the integrand electronic transition will provide further information about the electronic states [4]. The excitation of electrons from valence band to conduction band gives rise to the absorption coefficient $\alpha(\omega)$. Fundamental absorption edge is the change in absorption coefficient $\alpha(\omega)$ while the energy gap is the corresponding energy. The calculated data of direct and indirect band gap were tabulated and shown in Table 4, Figures 8(a), and 8(b). The indirect band gap is found to be in the range of $3.882-3.840 \mathrm{eV}$. It can be seen from the figure that the band gap of prepared glass samples decreases linearly with increasing $\mathrm{Er}^{3+} \mathrm{NPs}$ content. The change in band gap is due to the shifts of the valence and conduction band from each other.
TABLE 4: Indirect optical band gap $\left(E_{\text {opt }}^{1}\right)$, direct optical band gap $\left(E_{\text {opt }}^{2}\right.$ ), and Urbach energy $(\Delta E)$ of erbium NPs doped glass samples.

\begin{tabular}{lccc}
\hline Sample, $y$ & $E_{\mathrm{opt}}^{1}$ & $E_{\mathrm{opt}}^{2}$ & $\Delta E(\mathrm{eV})$ \\
\hline 0.000 & 3.280 & 3.600 & 0.185 \\
0.005 & 3.882 & 4.440 & 0.265 \\
0.010 & 3.876 & 4.390 & 0.270 \\
0.020 & 3.870 & 4.385 & 0.272 \\
0.030 & 3.860 & 4.380 & 0.273 \\
0.040 & 3.850 & 4.370 & 0.271 \\
0.050 & 3.840 & 4.360 & 0.276 \\
\hline
\end{tabular}

Previous research reported that particles size affects the band gap of the materials [14]. Small size of particles causes the width of both valence and conduction band to decrease. Therefore, the band gap will tend to become wider and results in the increasing value of band gap. Gupta and Ramrakhiani reported that the optical properties of materials highly depend on the size of particles [14]. The band gap increases as the size of particles decreases. It is obvious from the present glass samples that the value of band gap is much higher than that of the reported glass [15]. This is due to fact that the particles size is dependent on the optical properties of materials.

A plot of band gap, $E_{g}$, against concentration of erbium, $\mathrm{Er}_{2} \mathrm{O}_{3}$, shows that the band gap, $E_{g}$, linearly decreases with the increasing $\operatorname{Er}_{2} \mathrm{O}_{3}$ NPs content in the glass samples. This behavior can be explained by the structural changes in the glass system. It is well known that erbium and boron have more than one stable electronic configuration. As the content of erbium oxide, $\mathrm{Er}_{2} \mathrm{O}_{3}$ increases the bond length of $\mathrm{BO}_{3}$ structural units also increases. This is in accordance to the 


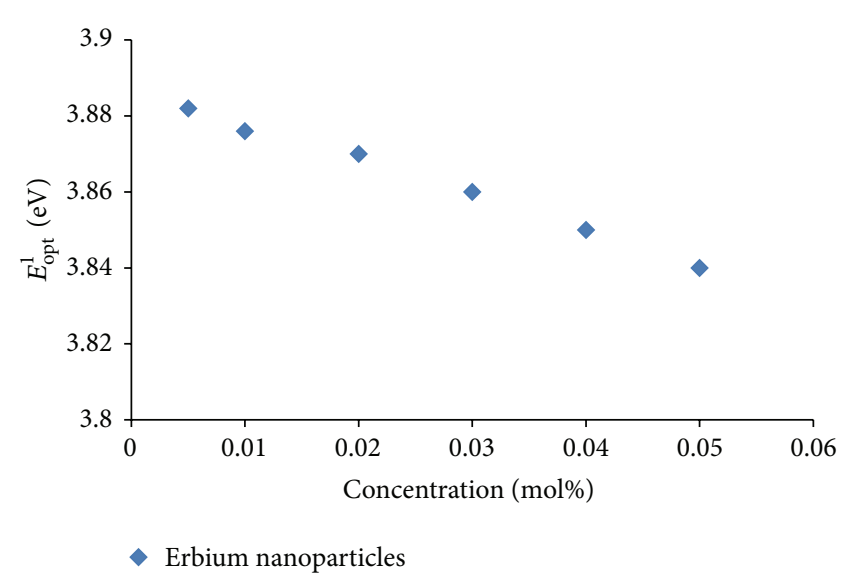

(a)

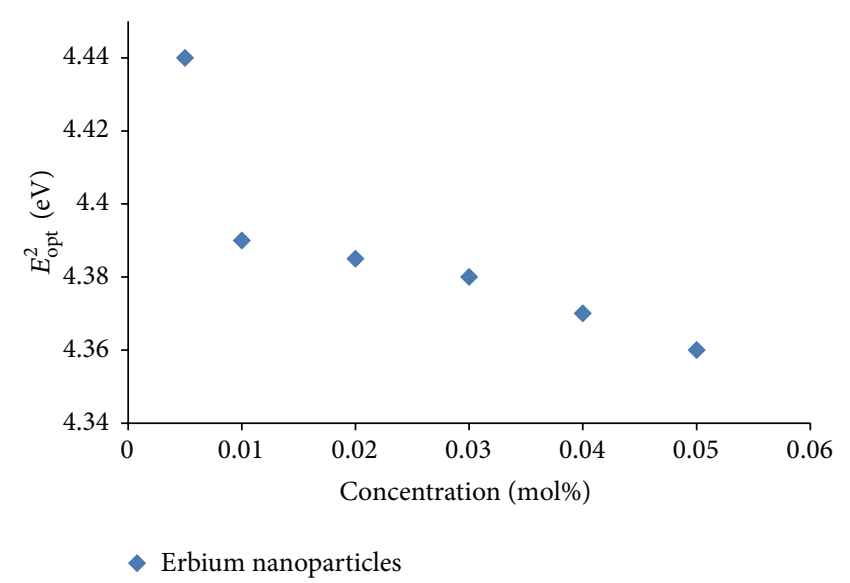

(b)

FIGURE 8: Optical band gap energy $\left(E_{\text {opt }}\right)$ and Urbach energy $(\Delta E)$. (a) Variation of indirect optical band gap with glass composition for indirect transition for erbium nanoparticles doped glass samples. (b) Variation of direct optical band gap with glass composition for direct transition erbium nanoparticles doped glass samples.

direct proportionality to the molar volume and the formation of NBO. These factors are responsible of the decreasing value of band gap energy in the glass system. Another possibility is the differences between the bond strength of erbium oxide and boron oxide. Erbium oxide is known to have weaker bond strength, Er-O $(147.0 \mathrm{kcal} / \mathrm{mol})$, compared to boron oxide, B-O $(193.3 \mathrm{kcal} / \mathrm{mol})$. This will contribute to the decreasing value of the band gap energy.

Urbach energy $(\Delta E)$ gives information regarding the character of disorder in the amorphous materials. It can be defined by a plot of natural logarithm of absorption coefficient $(\alpha(v))$ as a function of photon energy $(\hbar v)$ and determining of its slope as given by [16]

$$
\alpha(v)=\beta \exp \left(\frac{\hbar v}{\Delta E}\right),
$$

where $\beta$ is a constant, $\hbar$ is the plank constant, $v$ is the photon frequency, and $\Delta E$ is the Urbach energy which correspond to the width of localized states which is used to characterize the degree of disorder in the amorphous and crystalline materials [2]. The Urbach energy data for the prepared glass samples were tabulated and shown in Table 4. The Urbach energy values of the prepared glasses are found in the range 0.185$0.276 \mathrm{eV}$. It is known that materials which possess large value of Urbach energy have higher tendency to convert the weak bonds into defects. It can be seen that the Urbach energy increases as the content of erbium NPs oxide increases. This is probably due to the increase in the $\mathrm{TeO}_{4}$ pyramids as the content of erbium NPs oxide increases. The presence of $\mathrm{TeO}_{4}$ pyramids results in less stable structure and lower connectivity in the glass network.

\section{Conclusions}

Effects of erbium NPs on the optical properties of zinc borotellurite have been studied and investigated. The density of the prepared glass samples was found to be increased with an increase content of erbium NPs which is due to the formation of nonbridging oxygen. The molar volume is shown to be increased with an increase of erbium NPs. This is due to the larger value of erbium ionic radii than that of tellurite. The XRD analysis confirmed that all the glass samples are amorphous. The FTIR analysis consists of several bands which indicate the characteristic of $\mathrm{Te}-\mathrm{O}$ and $\mathrm{B}-\mathrm{O}$ vibrational groups. The presence of erbium leads to the formation of nonbridging oxygen and changes the ionic state into covalent bonds. The optical absorption spectra consist of several peaks positioned at 487.0; 521.5; $545.5 ; 652.0$; and $920.5 \mathrm{~nm}$ wavelengths. The absorption edge is found to be shifted to longer wavelength. The band gap of erbium NPs doped glass was found to be decreased linearly with an increases content of erbium NPs which is due to the formation of nonbridging oxygen in the glass system. From the result, the Urbach energy is found to be increased as the content of erbium NPs increase. This is due to the presence of $\mathrm{TeO}_{4}$ pyramids which leads the structure to become less stable. The information of the present study could give higher impact on designing photonic device by using erbium nanoparticles (NPs) doped zinc borotellurite glass system.

\section{Acknowledgment}

The authors appreciate the financial support for the work from the Ministry of Higher Education of Malaysia through RUGS (9199837).

\section{References}

[1] K. Selvaraju and K. Marimuthu, "Structural and spectroscopic studies on concentration dependent $\mathrm{Er}^{3+}$ doped boro-tellurite glasses," Journal of Luminescence, vol. 132, no. 5, pp. 1171-1178, 2012.

[2] K. Maheshvaran, P. Veeran, and K. Marimuthu, "Structural and optical studies on $\mathrm{Eu}^{3+}$ doped borotellurite glasses," Journal of Solid State Sciences, vol. 17, pp. 54-62, 2013. 
[3] P. G. Pavani, K. Sadhana, and V. C. Mouli, "Optical, physical and structural studies of boro-zinc tellurite glasses," Physica B, vol. 406, no. 6-7, pp. 1242-1247, 2011.

[4] M. Farouk, A. Samir, F. Metawe, and M. Elokr, "Optical absorption and structural studies of bismuth borate glasses containing $\mathrm{Er}^{3+}$ ions," Journal of Non-Crystalline Solids, vol. 371372, pp. 14-21, 2013.

[5] S. Rada, A. Dehelean, and E. Culea, "FTIR and UV-VIS spectroscopy investigations on the structure of the europiumlead-tellurate glasses," Journal of Non-Crystalline Solids, vol. 357, no. 16-17, pp. 3070-3073, 2011.

[6] G. W. Greenwood, "The growth of dispersed precipitates in solutions," Acta Metallurgica, vol. 4, no. 3, pp. 243-248, 1956.

[7] Y. B. Saddeek and M. S. Gaafar, "Physical and structural properties of some bismuth borate glasses," Materials Chemistry and Physics, vol. 115, no. 1, pp. 280-286, 2009.

[8] E. Yousef and B. Al-Qaisi, "UV spectroscopy, refractive indices and elastic properties of the $(76-x) \mathrm{TeO}_{2}$. $9 \mathrm{P}_{2} \mathrm{O}_{5} \cdot 15 \mathrm{ZnO} \cdot x \mathrm{LiNbO}_{3}$ glass," Journal of Solid State Sciences, vol. 19, pp. 6-11, 2013.

[9] R. El-Mallawany, M. D. Abdalla, and I. A. Ahmed, "New tellurite glass: optical properties," Materials Chemistry and Physics, vol. 109, no. 2-3, pp. 291-296, 2008.

[10] V. Dimitrov and T. Komatsu, "Classification of oxide glasses: a polarizability approach," Journal of Solid State Chemistry, vol. 178, no. 3, pp. 831-846, 2005.

[11] Y. Wang, S. Dai, F. Chen, T. Xu, and Q. Nie, "Physical properties and optical band gap of new tellurite glasses within the $\mathrm{TeO}_{2}-$ $\mathrm{Nb}_{2} \mathrm{O}_{5}-\mathrm{Bi}_{2} \mathrm{O}_{3}$ system," Materials Chemistry and Physics, vol. 113, no. 1, pp. 407-411, 2009.

[12] H. Aboud, H. Wagiran, and I. Hossain, "Effect of co-doped $\mathrm{SnO}_{2}$ nanoparticles on the optical properties of $\mathrm{Cu}$-doped lithium potassium borate glass," Journal of Materials Letters, vol. 85, pp. 21-24, 2012.

[13] N. Ahlawat, S. Sanghi, A. Agarwal, and S. Rani, "Effect of $\mathrm{Li}_{2} \mathrm{O}$ on structure and optical properties of lithium bismosilicate glasses," Journal of Alloys and Compounds, vol. 480, no. 2, pp. 516-520, 2009.

[14] P. Gupta and M. Ramrakhiani, "Influence of the particle size on the optical properties of CdSe nanoparticles," The Open Nanoscience Journal, vol. 3, pp. 15-19, 2009.

[15] Z. A. S. Mahraz M, M. R. Sahar, and S. K. Goshal, "Concentration dependent luminsence quenching of $\mathrm{Er}^{3+}$-doped zinc boro-tellurite glass," Journal of Luminescence, vol. 144, pp. 139145, 2013.

[16] M. D. Reza, M. R. Sahar, and S. K. Ghosal, "Effect of AgCl on spectroscopic properties of erbium doped zinc tellurite glass," Journal of Molecular Structure, vol. 1035, pp. 6-12, 2013. 

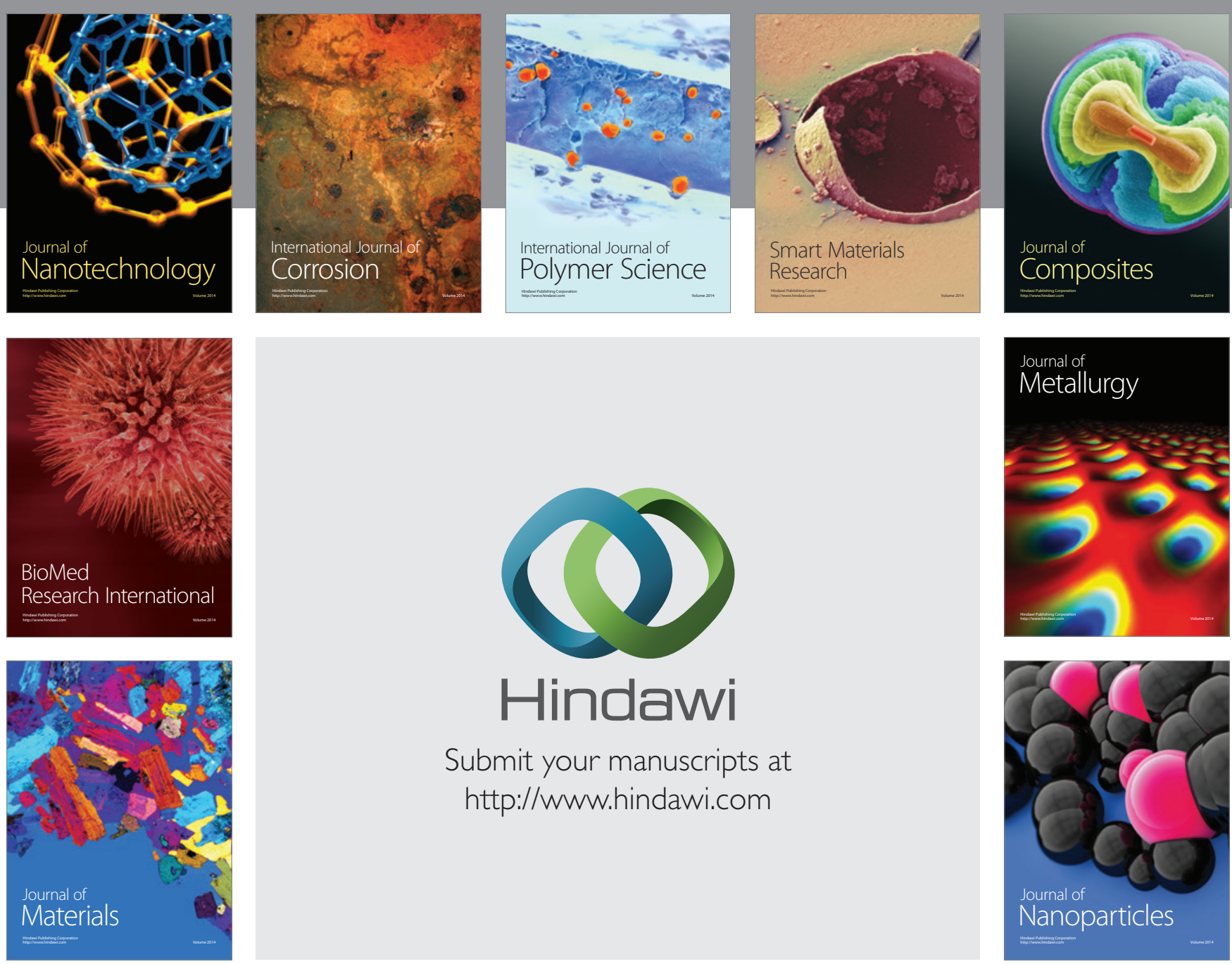

Submit your manuscripts at http://www.hindawi.com
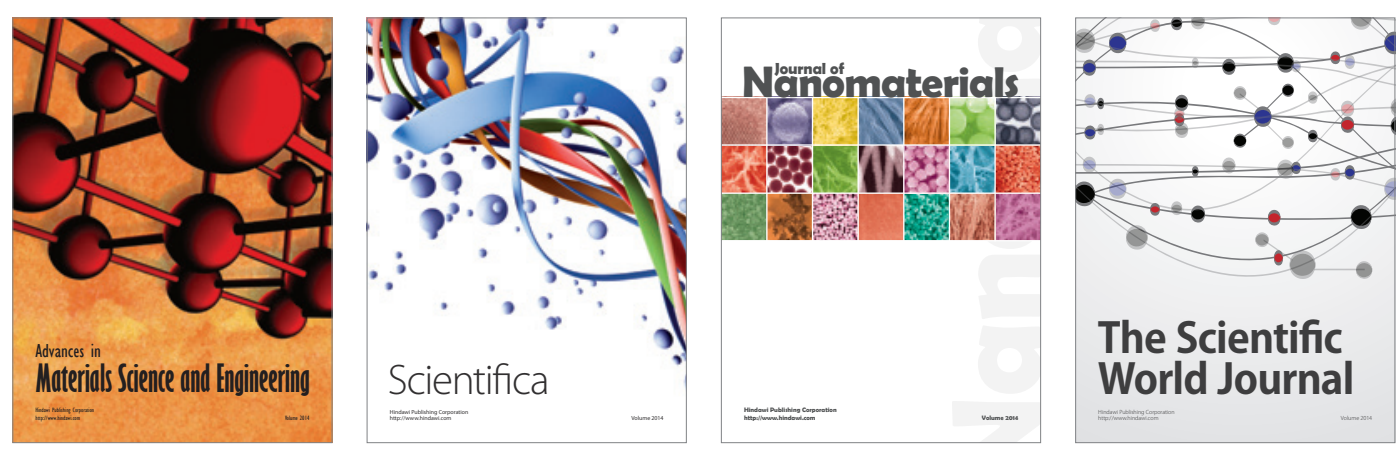

\section{The Scientific World Journal}
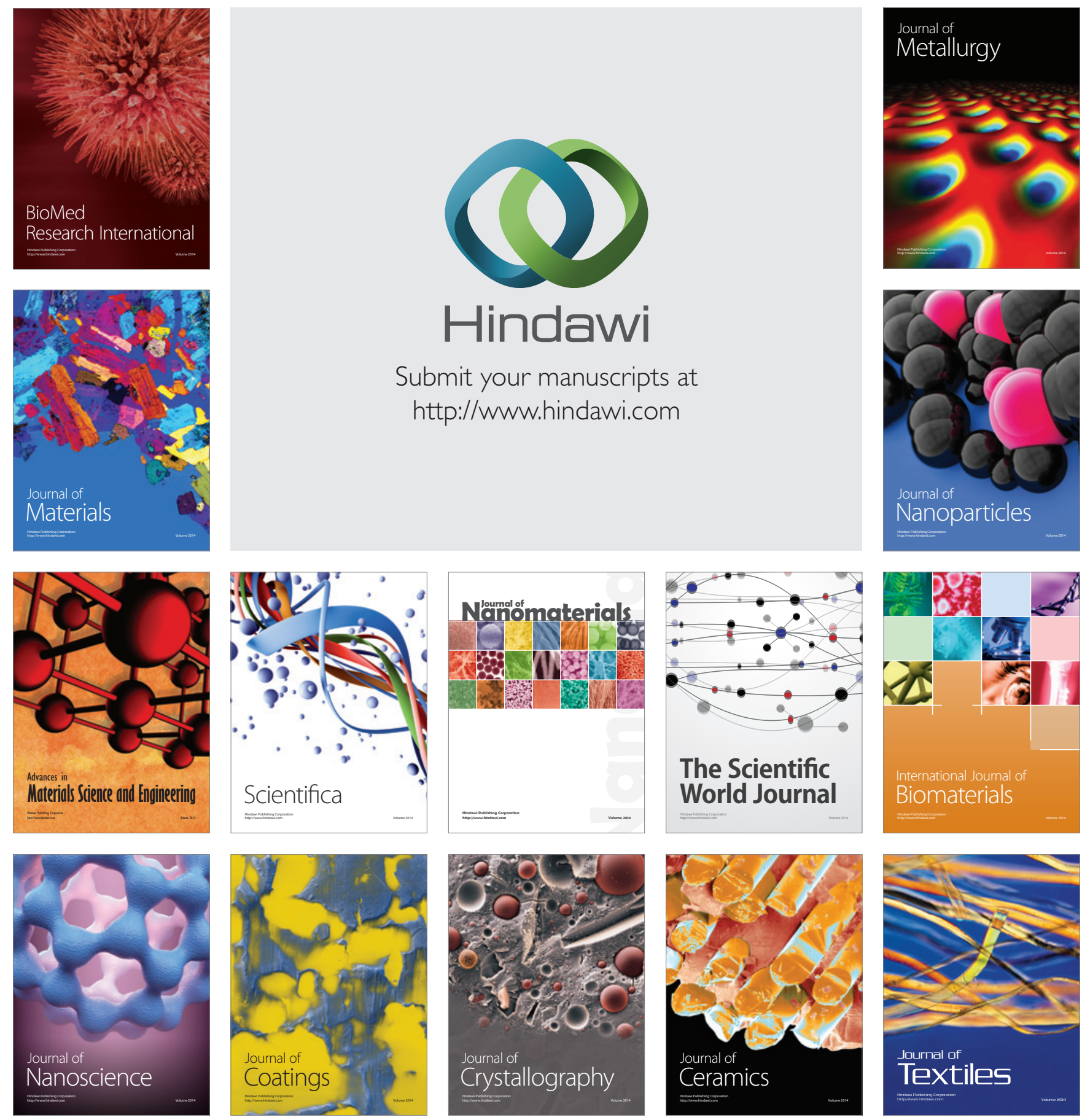